\title{
State-to-state rotational transitions in $\mathbf{H}_{2}+\mathbf{H}_{2}$ collisions at low temperatures
}

\author{
Teck-Ghee Lee \\ Department of Physics and Astronomy, \\ University of Kentucky, Lexington, KY 40506 \\ and Physics Division, Oak Ridge National Laboratory, Oak Ridge, TN 37831 \\ N. Balakrishnan \\ Department of Chemistry, University of Nevada Las Vegas, Las Vegas, Nevada 89154 \\ R. C. Forrey \\ Department of Physics, Penn State University, \\ Berks-Lehigh Valley College, Reading, PA 19610 \\ P. C. Stancil \\ Department of Physics and Astronomy and Center for Simulational Physics, \\ University of Georgia, Athens, GA 30602 \\ D. R. Schultz \\ Physics Division, Oak Ridge National Laboratory, Oak Ridge, TN 37831 \\ Gary J. Ferland \\ Department of Physics and Astronomy, \\ University of Kentucky, Lexington, KY 40506
}

(Dated: June 30, 2021) 


\begin{abstract}
We present quantum mechanical close-coupling calculations of collisions between two hydrogen molecules over a wide range of energies, extending from the ultracold limit to the super-thermal region. The two most recently published potential energy surfaces for the $\mathrm{H}_{2}-\mathrm{H}_{2}$ complex, the so-called DJ (Diep and Johnson, 2000) and BMKP (Boothroyd et al., 2002) surfaces, are quantitatively evaluated and compared through the investigation of rotational transitions in $\mathrm{H}_{2}+\mathrm{H}_{2}$ collisions within rigid rotor approximation. The BMKP surface is expected to be an improvement, approaching chemical accuracy, over all conformations of the potential energy surface compared to previous calculations of $\mathrm{H}_{2}-\mathrm{H}_{2}$ interaction. We found significant differences in rotational excitation/de-excitation cross sections computed on the two surfaces in collisions between two para$\mathrm{H}_{2}$ molecules. The discrepancy persists over a large range of energies from the ultracold regime to thermal energies and occurs for several low-lying initial rotational levels. Good agreement is found with experiment (Maté et al., 2005) for the lowest rotational excitation process, but only with the use of the DJ potential. Rate coefficients computed with the BMKP potential are an order of magnitude smaller.
\end{abstract}




\section{INTRODUCTION}

Collisions involving two hydrogen molecules are of great interest for three main reasons. First, the $\mathrm{H}_{2}+\mathrm{H}_{2}$ collision system is a prototype for chemical dynamics studies and can be used as a testing ground for scattering theory of inelastic (non-reactive) diatom-diatom collisions involving a weak interaction potential. Second, $\mathrm{H}_{2}$ is the most abundant molecular species in the universe. The rotational and vibrational transitions in $\mathrm{H}_{2}$ induced by collisions with its twin are of practical importance in models of astrophysical environments where the physical conditions may not be accessible to terrestrial experiments. Examples include low densities characteristic of giant molecular clouds in the interstellar medium where star formation occurs and $\mathrm{H}_{2}$ may act as a coolant ${ }^{1}$. Heating of the interstellar cloud by strong shock waves induces rotational and vibrational excitation of the $\mathrm{H}_{2}$ molecules leading to collision-induced dissociation to two free $\mathrm{H}$ atoms ${ }^{2}$ and photodissociation regions where $\mathrm{H}_{2}$ is exposed to strong UV stellar radiation ${ }^{3}$. Third, with recent experimental advances in the cooling and trapping of molecules ${ }^{4,5.6 .7}$ to form molecular Bose-Einstein condensates, collisional studies of the $\mathrm{H}_{2}+\mathrm{H}_{2}$ system can serve as a model to provide new insights into the behavior of diatom-diatom collisions at ultracold temperatures including investigations of Feshbach resonances, predissociation in van der Waals complexes, determination of complex scattering lengths, testing of effective range theory and Wigner threshold laws, and quasiresonant vibration-rotation energy transfer $8,9,10,11,12$ in molecular collisions.

Since $\left(\mathrm{H}_{2}\right)_{2}$ is the simplest 4-identical-particle closed shell system and there is a continuing demand for accurate collisional data for modeling astrophysical, atmospheric and

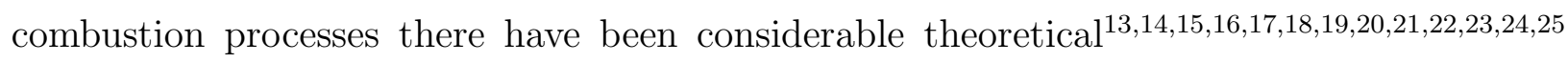

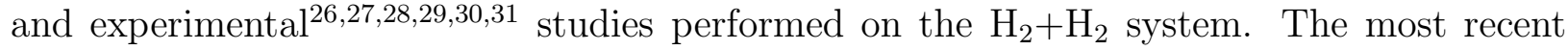
experiment was performed by Maté and co-workers ${ }^{31}$. Using the technique of Raman spectroscopy with supersonic expansions of para- $\mathrm{H}_{2}$, they measured the rate coefficient, $k_{00 \rightarrow 20}$, for $\mathrm{H}_{2}\left(j_{1}=0\right)+\mathrm{H}_{2}\left(j_{2}=0\right) \rightarrow \mathrm{H}_{2}\left(j_{1}^{\prime}=2\right)+\mathrm{H}_{2}\left(j_{2}^{\prime}=0\right)$ collisions in the temperature range of 2 to $110 \mathrm{~K}$. With their experimental methodology, without the loss of generality, the reduction of the measured $k_{00 \rightarrow 20}$ rate coefficient to the corresponding cross section, $\sigma_{00 \rightarrow 20}$ in the collision energy range of 360 to $600 \mathrm{~cm}^{-1}$ was made possible. To complement the experimental measurement, Maté et al.$^{31}$ also performed coupled channel quantum scattering calculations to determine the $\sigma_{00 \rightarrow 20}$ excitation cross section as a function of the collision 
energy. Good agreement was found between the experimentally-derived cross sections and theoretical results obtained using the rigid rotor potential energy surface (PES) developed by Diep and Johnson (DJ) ${ }^{32}$.

The most recent theoretical study of rotational excitation in $\mathrm{H}_{2}+\mathrm{H}_{2}$ collisions was carried out by Gatti and co-workers 33 . They employed the wave-packet propagation method in conjunction with the multiconfiguration time-dependent Hartree algorithm to compute rotational excitation cross sections for collision energies up to $1.2 \mathrm{eV}$ by a flux analysis of the interaction of the wave-packet with a complex absorbing potential. Gatti et al. compared their results with the wave packet calculations of Lin and Guo ${ }^{34}$ who employed the coupled-states (CS) approximation which neglects Coriolis coupling. They found that the CS approximation can lead to rather reliable predictions provided the calculations are performed at low collision energies and low rotational excitations. However, without the aid of accurate molecular potential curves, it is difficult to determine in which energy regime the Coriolis coupling takes effect for a specific collision system. Both wave-packet calculations employed the PES constructed in 2002 by Boothroyd, Martin, Keogh, and Peterson ${ }^{35}$ $(\mathrm{BMKP})$. While previous calculations ${ }^{18}$ have indicated that the CS approximation can give accurate results for rotational excitation in $\mathrm{H}_{2}$ at low energies, these calculations have used rather simple potential functions for the $\mathrm{H}_{4}$ system.

The purpose of this study is to perform accurate close-coupling calculations of rotational transitions in $\mathrm{H}_{2}+\mathrm{H}_{2}$ collisions within the ground vibrational state. Due to the relatively large vibrational level spacings of the $\mathrm{H}_{2}$ molecule and the weak $\mathrm{H}_{2}-\mathrm{H}_{2}$ interaction potential, the rigid rotor approximation is expected to hold well for the present system for pure rotational energy transfer. This is also confirmed by the wave packet calculations of Lin and $\mathrm{Guo}^{34}$. Here, we perform close-coupling calculations of rotational excitation on both the DJ and the BMKP PESs and compare our results with the wave-packet results of Lin and Guo and Gatti et al. So far, the accuracy of the BMKP PES has not been established by comparing results from accurate quantum calculations with experimental results. Such a comparison is provided here for both the DJ and BMKP PESs and we show that the rigid rotor DJ potential yield results that are generally in better agreement with experiment.

We also investigate the behavior of elastic and rotationally inelastic collisions in diatomdiatom collisions at ultracold energies by taking the $\mathrm{H}_{2}+\mathrm{H}_{2}$ system as an illustrative example. While ultracold rotational and vibrational energy transfer in atom-diatom collisions have 
extensively been reported ${ }^{8.9 .10 .11 .12 .36}$ in the last several years, such calculations are yet to be performed on molecule-molecule collisions. Forrey ${ }^{12}$ has performed limited calculations on $\mathrm{H}_{2}+\mathrm{H}_{2}$ collisions in the ultracold regime using the semi-empirical potential of Zarur and Rabitz ${ }^{16}$ while Avdeenkov and Bohn ${ }^{37,38.39}$ reported spin-exchange collisions in $\mathrm{O}_{2}+\mathrm{O}_{2}$ and $\mathrm{OH}+\mathrm{OH} / \mathrm{OD}+\mathrm{OD}$ systems. Here, we provide a detailed investigation of rotational energy transfer in $\mathrm{H}_{2}+\mathrm{H}_{2}$ collisions on the DJ and BMKP PESs at ultracold energies and report complex scattering lengths for collisions involving different initial rotational levels of the two molecules.

The paper is organized as follows: A brief description of the theoretical methodology is given in section II and results are presented in section [II] Section [V provides summary and conclusions. Atomic units are used throughout, unless otherwise noted: i.e., $\hbar=e=$ $m_{e}=a_{o}=1$ a.u., while 1 hartree $=27.2116 \mathrm{eV}=627.51 \mathrm{kcal} / \mathrm{mol}$.

\section{THEORY}

Calculations of state-to-state rotational transition cross sections and rate coefficients can provide an important test of the reliability of the potential energy surfaces describing the interaction of two $\mathrm{H}_{2}$ molecules when compared to available experiments. To compute the scattering amplitudes and hence the cross sections, we use well established quantum mechan-

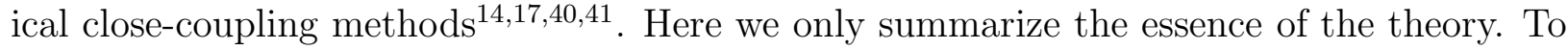
describe the scattering process, we solve the time-independent Schrödinger equation within rigid rotor approximation for the collision of two $\mathrm{H}_{2}$ molecules in the center of mass frame given by

$$
\left(\hat{T}(R)+\sum_{i=1}^{2} \hat{h}_{\text {rot }}\left(\hat{r}_{i}\right)+V\left(\vec{R}, \vec{r}_{1}, \vec{r}_{2}\right)-E\right) \Psi\left(\vec{R}, \hat{r}_{1}, \hat{r}_{2}\right)=0
$$

where $\hat{T}(R)=-\frac{1}{2 \mu} \nabla_{R}^{2}$ is the kinetic energy operator and $\hat{h}_{r o t}\left(\hat{r}_{i}\right)=\frac{\hat{j}_{i}}{2 \mu_{i} r_{i}^{2}}$ is the diatom rotational kinetic energy operator; $\mu$ and $\mu_{i}$ are the reduced masses of the $\mathrm{H}_{2}$ collision pair and a isolated $\mathrm{H}_{2}$ molecule, respectively. The internuclear distance between the two $\mathrm{H}$ atoms is denoted by $r_{i}$, and $R$ is the distance between the center of mass of the diatoms; $\hat{r}_{1}$ and $\hat{r}_{2}$ are the orientation angles of the rotors 1 and 2 , respectively. The term $V\left(\vec{R}, \vec{r}_{1}, \vec{r}_{2}\right)$ represents the $\mathrm{H}_{2}-\mathrm{H}_{2}$ interaction potential. 
The rotational part of the Hamiltonian operator satisfies the eigenvalue equation

$$
\left(\hat{h}_{\text {rot }}\left(\hat{r}_{i}\right)-B_{i} j_{i}\left(j_{i}+1\right)\right) Y_{j_{i} m_{i}}\left(\hat{r}_{i}\right)=0
$$

where $B_{i}$ are the rotational constants of the rigid rotors.

The rotational angular momenta of the two molecules $\vec{j}_{1}$ and $\vec{j}_{2}$ are coupled to form $\vec{j}_{12}$, which is subsequently coupled to the orbital momentum $\vec{l}$ to yield the total angular momentum $\vec{J}$. The angular wave function in the total angular momentum representation is given by

$$
\begin{aligned}
\phi_{j_{1} j_{2} j_{12} l}^{J M}\left(\hat{R}, \hat{r}_{1}, \hat{r}_{2}\right)= & \sum_{m_{1} m_{2} m_{12} m_{l}}\left(j_{1} m_{1} j_{2} m_{2} \mid j_{12} m_{12}\right)\left(j_{12} m_{12} l m_{l} \mid J M\right) \\
& \times Y_{j_{1} m_{1}}\left(\hat{r}_{1}\right) Y_{j_{2} m_{2}}\left(\hat{r}_{2}\right) Y_{l m_{l}}(\hat{R})
\end{aligned}
$$

and under spatial inversion

$$
\mathcal{P} \phi_{\alpha l}^{J M}\left(\hat{R}, \hat{r}_{1}, \hat{r}_{2}\right)=(-1)^{j_{1}+j_{2}+j_{12}} \phi_{\alpha l}^{J M}\left(\hat{R}, \hat{r}_{1}, \hat{r}_{2}\right)
$$

where $\alpha \equiv j_{1} j_{2} j_{12}$, and $m_{1}, m_{2}, m_{12}$ and $m_{l}$ are the projections of $\vec{j}_{1}, \vec{j}_{2}, \vec{j}_{12}$ and $\vec{l}$, respectively, onto the space-fixed $z$-axis. The symbol $\left(j_{1} j_{2} m_{1} m_{2} \mid J M\right)$ denotes a Clebsch-Gordon coefficient.

Using the basis set expansion method, we expand our total wave function ansatz as

$$
\Psi\left(\vec{R}, \hat{r}_{1}, \hat{r}_{2}\right)=\frac{1}{R} \sum_{J M \alpha l} F_{\alpha l}^{J M}(R) \phi_{\alpha l}^{J M}\left(\hat{R}, \hat{r}_{1}, \hat{r}_{2}\right)
$$

Substituting eqn.(15) into eqn.(11), we arrive at a system of close-coupling equations

$$
\left(\frac{d^{2}}{d R^{2}}-\frac{l(l+1)}{R^{2}}+2 \mu E_{k}\right) F_{\alpha l}^{J}(R)=2 \mu \sum_{\alpha^{\prime} l^{\prime}} F_{\alpha^{\prime} l^{\prime}}^{J}(R)\left\langle\phi_{\alpha l}^{J M}|V| \phi_{\alpha^{\prime} l^{\prime}}^{J M}\right\rangle,
$$

where the quantities in $\langle\cdots\rangle$ are the matrix elements of the interaction potential and $E_{k}=$ $E-B_{1} j_{1}\left(j_{1}+1\right)-B_{2} j_{2}\left(j_{2}+1\right)$ is the kinetic energy of the relative motion for a given value of the total energy $E$. The solution of the coupled equations and asymptotic analysis of the radial wave functions yield the scattering S-matrix from which cross sections for state-tostate rotational transitions from an initial level specified by quantum numbers $j_{1} j_{2}$ to final levels $j_{1}^{\prime} j_{2}^{\prime}$ are given by

$$
\sigma_{j_{1} j_{2} \rightarrow j_{1}^{\prime} j_{2}^{\prime}}\left(E_{k}\right)=\frac{\pi}{2 \mu E_{k}\left(2 j_{1}+1\right)\left(2 j_{2}+1\right)} \sum_{J j_{12} j_{12}^{\prime} l l^{\prime}}(2 J+1)\left|\delta_{\nu \nu^{\prime}}-\mathcal{S}_{\nu \nu^{\prime}}^{J}\right|^{2}
$$


where $\nu \equiv j_{1} j_{2} j_{12} l$.

The above expression for cross section assumes that the two diatomic molecules are distinguishable. However, for $\mathrm{H}_{2}-\mathrm{H}_{2}$ collisions the target and projectile molecules are indistinguishable and one must take into account the symmetry of the wave function under exchange. Thus, properly symmetrized total angular momentum wave functions $\frac{17}{}$

$$
\begin{aligned}
\phi_{j_{1} j_{2} j_{12} l}^{J M \pm}\left(\hat{R}, \hat{r}_{1}, \hat{r}_{2}\right)= & \frac{1}{\sqrt{2\left(1+\delta_{j_{1} j_{2}}\right)}}\left[\phi_{j_{1} j_{2} j_{12} l}^{J M}\left(\hat{R}, \hat{r}_{1}, \hat{r}_{2}\right)\right. \\
& \left. \pm(-1)^{j_{1}+j_{2}+j_{12}+l} \phi_{j_{1} j_{2} j_{12} l}^{J M}\left(\hat{R}, \hat{r}_{1}, \hat{r}_{2}\right)\right]
\end{aligned}
$$

need to be employed in which the index pair $j_{1} j_{2}$ is restricted to $j_{1} \geq j_{2}$ to obtain a linearly independent set. Using the symmetrized angular wave functions, one obtains coupled equations similar to eqn. (6) which yield scattering cross sections ${ }^{17}$

$$
\sigma_{j_{1} j_{2} \rightarrow j_{1}^{\prime} j_{2}^{\prime}}\left(E_{k}\right)=\frac{\pi\left(1+\delta_{j_{1} j_{2}}\right)\left(1+\delta_{j_{1}^{\prime} j_{2}^{\prime}}\right)}{2 \mu E_{k}\left(2 j_{1}+1\right)\left(2 j_{2}+1\right)} \sum_{J j_{12} j_{12}^{\prime} l l^{\prime}}(2 J+1)\left|\delta_{\nu \nu^{\prime}}-\mathcal{S}_{\nu \nu^{\prime}}^{J}\right|^{2} .
$$

Rate coefficients for state-to-state rotational transitions are obtained by averaging the appropriate cross sections over a Boltzmann distribution of relative speeds of the projectile molecule at a given temperature $T$ :

$$
k_{j_{1} j_{2} \rightarrow j_{1}^{\prime} j_{2}^{\prime}}(T)=G \int_{0}^{\infty} d E_{k} \sigma_{j_{1} j_{2} \rightarrow j_{1}^{\prime} j_{2}^{\prime}}\left(E_{k}\right) E_{k} e^{\left(-\beta E_{k}\right)},
$$

where the constant $G=\sqrt{\frac{8}{\mu \pi \beta}} \beta^{2}$ and $\beta=\left(k_{B} T\right)^{-1}$ with $k_{B}$ being the Boltzmann constant. The total quenching rate coefficient can be calculated from

$$
k_{j_{1} j_{2}}(T)=\sum_{j_{1}^{\prime} j_{2}^{\prime}} k_{j_{1} j_{2} \rightarrow j_{1}^{\prime} j_{2}^{\prime}}(T)
$$

\section{RESULTS}

We have carried out close-coupling calculations for collisions of $\mathrm{H}_{2}$ with $\mathrm{H}_{2}$ using the BMKP and DJ PESs. The rigid rotor target and projectile energy levels were calculated using a rotational constant of $B=60.853 \mathrm{~cm}^{-1}$ for the $\mathrm{H}_{2}$ molecule. To solve the coupled radial equations (6), we used the hybrid modified log-derivative-Airy propagator ${ }^{43}$ in the general purpose non-reactive scattering code MOLSCAT $\stackrel{44}{ }$. The log-derivative matrix ${ }^{43}$ is propagated to large intermolecular separations where the numerical results are matched to 
the known asymptotic solutions to extract the physical scattering matrix. This procedure is carried out for each partial wave until a converged cross section is reached. We have checked that the results are converged with respect to the number of partial waves as well as the matching radius for all channels included in the calculations.

In addition to the partial wave convergence, based on the DJ PES, we have checked that the results are converged with respect to various parameters that enter into the close-coupling calculations. These include the number of quadrature points used for angular integration, the number of terms in the angular expansion of interaction potential, and the asymptotic matching radius for radial integration. In the ultracold regime, we used a matching radius of $R_{o}=200-300$ a.u. to obtain converged values of elastic and inelastic cross sections while for the subthermal energy region, a matching radius of $50 \mathrm{a} . \mathrm{u}$. was sufficient to yield results of comparable accuracy. Similarly, we used 10 quadrature points each for integration along angular coordinates $\theta_{1}, \theta_{2}$, and $\phi_{12}$.

Finally, two different basis sets (22-state: $j_{1} j_{2}=00,20,22, \cdots, 44$ and 50-state: $j_{1} j_{2}$ $=00,20,22, \cdots, 66)$ were also employed to further test the convergence of our results. For $E<1.0 \mathrm{eV}$, the two basis sets yield results within $1 \%$, and at $E=1.0 \mathrm{eV}$, a similar degree of accuracy was obtained for the dominant transitions. For weaker transitions such as 00 $\rightarrow 44$ rotational excitation, the small and large basis sets gave $\sigma_{00 \rightarrow 44}=3.01 \times 10^{-18}$ and $3.20 \times 10^{-18} \mathrm{~cm}^{2}$, respectively, at $1.0 \mathrm{eV}$. Note that the cross sections for dominant transitions are two orders of magnitude larger than the weaker ones. Even at $E=2.6 \mathrm{eV}$, the cross sections obtained from both basis sets for the dominant transitions have similar convergence properties as for $E=1.0 \mathrm{eV}$. However, for $00 \rightarrow 44$, we found that the larger basis set is preferred since there is a significant difference between the cross sections (i.e., $\sigma_{00 \rightarrow 44}=$ $4.31 \times 10^{-17} \mathrm{~cm}^{2}$ and $6.30 \times 10^{-17} \mathrm{~cm}^{2}$, respectively, from the small and large basis sets, at $2.6 \mathrm{eV}$ ). Since our focus is on the low-energy region where the two basis sets yield similar results, the smaller basis set is adopted throughout the calculations.

Fig. 1 shows the comparison between theoretical and experimental rate coefficients for the $00 \rightarrow 20$ transition in the temperature range between $50 \mathrm{~K}$ and $300 \mathrm{~K}$. Both experimental and theoretical rate coefficients indicate a precipitous drop for temperatures lower than $100 \mathrm{~K}$. Unexpectedly, we find that the $00 \rightarrow 20$ excitation rate coefficient computed with the BMKP PES is about an order of magnitude smaller than that calculated with the PES of DJ and the experiment, though both BMKP and DJ display the same trend. Only results from the DJ 


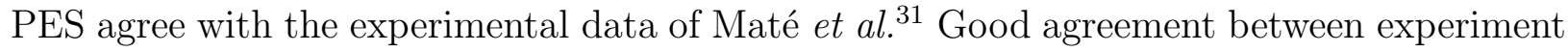
and theory based on the DJ PES was also shown by Maté et al. ${ }^{31}$. Further, the theoretical results obtained by Flower ${ }^{21}$ using the older PES of Schwenke ${ }^{45}$ are also seen to be in good agreement with experiment. The discrepancy with the BMKP results may directly be traced to the weaker anisotropy of the BMKP PES responsible for the $00 \rightarrow 20$ transition. In the calculations the angular dependence of the interaction potential is represented $\mathrm{as}^{32}$

$$
V\left(R, \theta_{1}, \theta_{2}, \phi_{12}\right)=\sum_{l_{1}, l_{2}, l} V_{l_{1}, l_{2}, l}(R) G_{l_{1}, l_{2}, l}\left(\theta_{1}, \theta_{2}, \phi_{12}\right)
$$

where $V_{l_{1}, l_{2}, l}(R)$ are radial expansion coefficients and $G_{l_{1}, l_{2}, l}\left(\theta_{1}, \theta_{2}, \phi_{12}\right)$ are bispherical harmonics. In Fig. 2 we compare the spherically symmetric $\left(V_{000}\right)$ and the leading anisotropic terms, $V_{022}=V_{202}$ and $V_{224}$, in the angular expansion of the BMKP and DJ interaction potentials as functions of the intermolecular separation. It is seen that while the spherically symmetric part is nearly identical for both potentials, the main anisotropic term, $V_{022}=V_{202}$, responsible for the $00 \rightarrow 20$ rotational excitation is smaller for the BMKP potential at small intermolecular separations. Our test calculations show that at energies lower than $0.1 \mathrm{eV}$, the discrepancy between the two results is mostly due to small differences in the coupling elements in the region of the van der Waals minimun, i.e., $R>3.0$ au. The same also applies to the next higher order term, $V_{224}$. On the other hand, Progrebnya and Clary $\stackrel{46}{4}$ found that the BMKP surface yields too high values for vibrational relaxation in $\mathrm{H}_{2}(v=1)+\mathrm{H}_{2}(v=0)$ collisions. They attributed this to higher-order anisotropic terms in the BMKP PES that leads to preferential population of high rotational levels in the $v^{\prime}=0$ level after quenching (see Fig. 2 of the above reference). Calculations employing a modified version of the BMKP potential in which only the first two leading anisotropic terms of the interaction potential shown in Fig. 2 are retained gave results in better agreement with the experiment.

The integral elastic cross sections of para- $\mathrm{H}_{2}+$ para- $\mathrm{H}_{2}$ collisions as a function of collision energy is plotted in Fig. 3(a) for both the BMKP and DJ potentials. In the zero-energy limit, the elastic cross sections attain finite values in accordance with Wigner's law. The limiting value of the elastic cross section is $1.91 \times 10^{-13} \mathrm{~cm}^{2}$ and $1.74 \times 10^{-13} \mathrm{~cm}^{2}$ for the BMKP and DJ PESs, respectively. The comparable values of the limiting elastic cross sections on the two potentials is explained based on the nearly identical values for the spherically symmetric part of the interaction potentials for both surfaces (see Fig. 2). Both potentials exhibit a shape resonance at collision energies between 0.0002 and $0.0003 \mathrm{eV}$, arising from the $l=2$ 
partial wave. At higher energies, cross sections on the two potentials exhibit an oscillatory behaviour (see inset in the top panel of Fig. 3) which arises from interference between partial cross sections corresponding to different values of the total angular momentum quantum number $J$. Note that only even values of $J$ are allowed for $\mathrm{p}-\mathrm{H}_{2}-\mathrm{p}-\mathrm{H}_{2}$ collisions. Schaefer and Meyer ${ }^{19}$ have provided a detailed analysis of the oscillatory behavior of the elastic cross sections.

In Fig. 3(b) we compare elastic cross sections from the present work on the DJ and BMKP surfaces with the theoretical results of Diep and Johnson ${ }^{32}$ and the experimental measurement of Bauer et al. $\stackrel{47}{ }$. It is seen that the results on the DJ potential give slightly better agreement with experimental data although the overall agreement between the experiment and theory is generally good.

In Fig. 4 we compare cross sections from the present calculations on the BMKP surface for the $00 \rightarrow 20,00 \rightarrow 22$ and $00 \rightarrow 40$ transitions with the wave packet results of Gatti et al. ${ }^{33}$ and Lin and Guo $\stackrel{34}{ }$. The overall agreement between the CC and the wave-packet results is rather good, to within $10-15 \%$ for all cross sections, except for the $00 \rightarrow 22$ rotational excitation for which the present results are 30-50\% larger. The significant differences between the present results and the wave packet results for this transition is somewhat surprising considering the fact that the corresponding cross sections are about an order of magnitude larger than the $00 \rightarrow 40$ transition for which we obtain good agreement with the wave packet results. We are confident that this is not a numerical error in our calculations as we have benchmarked our results against a new time-independent coupled channel code developed by Krems ${ }^{48}$ which reproduces our results up to several significant digits for all transitions shown in Fig. 4. The deviation of the CS wave-packet results of Lin and Guo from the present $\mathrm{CC}$ and full wave-packet data of Gatti et al. at higher energies may be attributed to Coriolis couplings. This evidently suggests that Coriolis coupling plays an important role at higher energies and that the rigid rotor approximation appears to hold well for this system for collision energies investigated in the present work.

In Fig. 5(a), we compare rotational quenching cross sections for the $20 \rightarrow 00$ transition evaluated using the BMKP and DJ potential with the $20 \rightarrow 20$ elastic scattering cross section. Since the elastic scattering cross section on the two PESs is comparable, only the result on the DJ potential is shown. The resonance feature that occurs in all three cross sections just above $10^{-4} \mathrm{eV}$ is due the $l=2$ shape resonance discussed previously (See Fig. 
1). It is seen that the inelastic cross sections are much smaller than the elastic one at all energies shown in the Fig. 5(a). This suggests that it may be possible to cool rotationally excited $\mathrm{H}_{2}$ molecules in the $j=2$ rotational level by thermalizing collisions with ground state $\mathrm{H}_{2}$ molecules (evaporative cooling) without significant trap loss, though the absence of an electric dipole moment makes it a difficult system to handle experimentally. For incident energies lower than $10^{-5} \mathrm{eV}$, the quenching cross section varies inversely with the velocity in accordance with Wigner threshold behavior. As a consequence, the product of the relative velocity and the quenching cross section attains a finite value in the limit of zero incident kinetic energy, as illustrated in Fig. 5(b). The limiting value of the quenching rate coefficient is $2.4 \times 10^{-13} \mathrm{~cm}^{3} / \mathrm{s}$.

In ultracold collisions where $s$-wave scattering dominates, elastic and inelastic scattering cross sections are conveniently expressed in terms of scattering lengths. The scattering length is real when only elastic scattering is present, but it becomes complex with the inclusion of inelastic channels 9 . The complex scattering length is given by $a_{j_{1} j_{2}}=\alpha_{j_{1} j_{2}}-i \beta_{j_{1} j_{2}}$ where $\alpha$ and $\beta$ are real and imaginary parts of the scattering length. The imaginary part of the scattering length $\beta$ is related to the zero-temperature limit of the quenching rate coefficient: $k_{j_{1} j_{2}}(T \rightarrow 0)=4 \pi \beta_{j_{1} j_{2}} \hbar / \mu$. For the DJ potential we obtain the values $\alpha_{00}=5.88 \AA$, $\alpha_{20}=5.78 \AA, \beta_{20}=0.003 \AA, \alpha_{22}=5.83 \AA$, and $\beta_{22}=0.0023 \AA$. The corresponding values for scattering on the BMKP potential are $\alpha_{00}=6.16 \AA, \alpha_{20}=6.16 \AA, \beta_{20}=0.00028 \AA$, $\alpha_{22}=6.16 \AA$, and $\beta_{22}=0.00071 \AA$. It is seen that the real part of the scattering length remains practically unchanged for the three initial states indicating that no zero-energy resonances occur for any of the three initial states on either PES. The presence of zero-energy resonances (bound/quasibound states near channel thresholds) generally enhances the elastic scattering cross section at low energies. The smaller value of the inelastic quenching rates on the BMKP surface is also reflected in the values of $\beta$ for all three initial states.

In Fig. 6 we compare cross sections for $22 \rightarrow 00$ and $22 \rightarrow 20$ transitions obtained using the DJ potential with the CC calculations of Forrey $\underline{\underline{12}}$ based on the PES of Zarur and Rabitz ${ }^{16}$. The agreement is remarkably good considering that the DJ potential is derived from accurate ab initio calculations while that of Zarur and Rabitz is a model semi-empirical potential. The reproduction of the shape resonance near $E=10^{-4} \mathrm{eV}$ by the two potentials is a good indicator of the accuracy of the two potential surfaces. The solid curve in Fig. 6 is the quenching cross section for $20 \rightarrow 00$ transition on the DJ potential and it is seen that 
the cross sections for $22 \rightarrow 20$ transition is larger at all energies.

Further comparison between BMKP and the DJ potential is presented in Fig. 7 in which we provide energy dependence of the excitation cross sections for $00 \rightarrow 20,22,40,42$ and 44 transitions. The general trend in all cases, except for $00 \rightarrow 40$ at low energies, is that the BMKP potential yields smaller values of cross sections compared to the DJ potential. The differences get somewhat smeared out when the cross sections are integrated over a Boltzmann distribution of relative velocities of the two molecules to yield the rate constants. This is illustrated in Fig. 8 for the same transitions as given in Fig. 7. Comparison is also made to the rate coefficients computed by Flower ${ }^{21}$ which show better agreement with the DJ results.

\section{SUMMARY AND CONCLUSIONS}

We have performed quantum close-coupling calculations of elastic and inelastic rotational transitions in collisions of $\mathrm{H}_{2}$ with $\mathrm{H}_{2}$ using the two most recently published ab initio interaction potential energy surfaces $(\mathrm{PESs})$ for the $\mathrm{H}_{4}$ system. The calculations span a wide range of energies (9-orders of magnitude) extending from the zero-temperature limit to about 2.0 $\mathrm{eV}$. Sensitivity of the results to details of the interaction potential is presented by computing real and imaginary parts of the scattering lengths for different initial rotational levels of the two colliding $\mathrm{H}_{2}$ molecules. It is shown that the limiting elastic cross section is not very sensitive to the initial rotational levels of the two $\mathrm{H}_{2}$ molecules although the inelastic cross sections strongly depend on the initial rotational level.

We also showed that results obtained using the rigid rotor potential surface of Diep and Johnson $^{32}$ are in close agreement with the experimental measurements of Maté et al. 1ㅜ $_{\text {for }}$ $00 \rightarrow 20$ rotational excitation rate coefficient. However, the corresponding results obtained using the Boothroyd et al. ${ }^{35}$ PES are a factor of ten smaller. This is quite significant because the Boothroyd et al. surface is generally believed to be the most accurate potential surface for the $\mathrm{H}_{4}$ system and it has been used in two most recent six-dimensional quantum mechanical calculations ${ }^{33.34}$ of rotational excitation in $\mathrm{H}_{2}+\mathrm{H}_{2}$ collisions. The present study demonstrates that the BMKP surface will need to be reevaluated before it can be adopted in large-scale scattering calculations, especially for astrophysical applications. 


\section{ACKNOWLEDGMENTS}

TGL and GJF acknowledges support from NASA grant NNG05GD81G and the Spitzer Space Telescope Theoretical Research Program. The work of RCF was supported by NSF grants PHY-0244066 and PHY-0554794. NB acknowledges support from NSF grant PHY0555565 and DOE grant DE-FG36-05GO85028. PCS acknowledges support from NSF grant AST-0087172. We acknowledge support from the Institute for Theoretical Atomic, Molecular, and Optical Physics at the Harvard-Smithsonian Center for Astrophysics for a workshop which initiated this work.

1 J. Le Boulot, G. Pineau des Forêts, and D. R. Flower, Mon. Not. Roy. Astron. Soc. 305, 802 (1999).

2 C. A. Chang and P. G. Martin, Astrophys. J. 378, 202 (1991).

3 G. Shaw, G. J. Ferland, N. P. Abel, P. C. Stancil, and P. A. M. van Hoof, Astrophys. J. 624, $794(2005)$.

4 J. M. Doyle, B. Friedrich, J. Kim, and D. Patterson, Phys. Rev. A 52, 3224 (1995); J. D. Weinsein, R. deCarvalho, T. Guillet, B. Friedrich, and J. M. Doyle, Nature (London) 395, 148 (1998).

5 J. T. Bahns, W. C. Stwalley, and P. L. Gould, J. Chem. Phys. 104, 9689 (1996); J. T. Bahns, P. L. Gould, and W. C. Stwalley, Adv. At. Mol. Opt. Phys. 42, 171 (2000).

6 A. Fioretti, D. Comparat, A. Crubellier, O. Dulieu, F. Masnou-Seeuws, and P. Pillet, Phys. Rev. Lett. 80, 4402 ? (1998).

7 T. Takekoshi, B. M. Patterson, and R. J. Knize, Phys. Rev. A. 59, R5 (1999).

8 N. Balakrishnan, R. C. Forrey, and A. Dalgarno, Chem. Phys. Lett. 280, 1 (1997); N. Balakrishnan, V. Kharchenko, R. C. Forrey, and A. Dalgarno, Chem. Phys. Lett. 280, 5 (1997).

9 N. Balakrishnan, R. C. Forrey, and A. Dalgarno, Phys. Rev. Lett. 80, 3224 (1998).

10 R. C. Forrey, N. Balakrishnan, V. Kharchenko, and A. Dalgarno, Phys. Rev. A. 58, R2645 (1998); R. C. Forrey, V. Kharchenko, N. Balakrishnan, and A. Dalgarno, Phys. Rev. A. 59, $2146(1999)$.

11 R. C. Forrey, N. Balakrishnan, A. Dalgarno, M. R. Haggerty, and E. J. Heller, Phys. Rev. Lett. 
82, 2657 (1999); R. C. Forrey, N. Balakrishnan, A. Dalgarno, M. R. Haggerty, and E. J. Heller, Phys. Rev. A. 64, 022706 (2001).

12 R. C. Forrey, Phys. Rev. A. 63, 051403(R) (2001); R. C. Forrey, Phys. Rev. A. 66, 023411 (2002).

13 W. D. Davison, Discuss. Faraday Soc. 33, 71 (1962).

14 K. Takayangi, Adv. At. Mol. Phys. 1, 149 (1965).

15 A. C. Allison and A. Dalgarno, Proc. Phys. Soc. 90, 609 (1967).

16 G. Zarur and H. Rabitz, J. Chem. Phys. 60, 2057 (1974).

17 S. Green, J. Chem. Phys. 62, 2271 (1975).

18 T. G. Heil, S. Green, and D. J. Kouri, J. Chem. Phys. 68, 2562 (1978).

19 J. Schaefer and W. Meyer, J. Chem. Phys. 70, 344 (1979).

20 G. Danby, D. R. Flower, and T. S. Monteiro, Mon. Not. R. Astron. Soc. 226, 739 (1987).

21 D. R. Flower, Mon. Not. R. Astron. Soc. 297, 334 (1998).

22 D. R. Flower and E. Roueff, J. Phys. B 31, 2935 (1998).

23 D. R. Flower and E. Roueff, J. Phys. B 32, 3399 (1999).

24 D. R. Flower, J. Phys. B 33, L193 (2000).

25 D. R. Flower, J. Phys. B 33, 5243 (2000). [ChemPort]

26 M. G. Dondi, U. Valbusa, and G. Scoles, Chem. Phys. Lett. 17, 137 (1972).

27 J. M. Farrar and Y. T. Lee, J. Chem. Phys. 57, 5492 (1972).

28 M. M. Audibert, C. Joffrin, and J. Ducuing, Chem. Phys. Lett. 25, 158 (1974); M.-M. Audibert, R. Vilaseca, J. Lukasik, and J. Ducuing, Chem. Phys. Lett. 31, 232 (1975).

29 J. E. Dove and H. Teitelbaum, Chem. Phys. 6, 431 (1974); J. E. Dove and H. Teitelbaum, Chem. Phys. 40, 87 (1979).

30 T. G. Kreutz, J. Gelfand, R. B. Miles, and H. Rabitz, Chem. Phys. 124, 359 (1988).

31 B. Maté, F. Thibault, G. Tejeda, J. M. Fernndez, and S. Montero, J. Chem. Phys. 122, 064313 (2005).

32 P. Diep and J. K. Johnson, J. Chem. Phys. 112, 4465 (2000); 1123, 3480 (2000)

33 F. Gatti, F. Otto, S. Sukiasyan, and Hans-Dieter Meyer, J. Chem. Phys. 123, 174311 (2005)

34 S. Y. Lin and H. Guo, J. Chem. Phys. 117, 5183 (2002).

35 A. I. Boothroyd, P. G. Martin, W. J. Keogh, and M. J. Peterson J. Chem. Phys. 116, 666 (2002);A. I. Boothroyd, W. J. Keogh, P. G. Martin, and M. R. Peterson, J. Chem. Phys. 95, 
4343 (1991).

36 N. Balakrishnan, A. Dalgarno, and R. C. Forrey, J. Chem. Phys. 113, 621 (2000).

37 A. V. Avdeenkov J. L. and Bohn, Phys. Rev. A 64, 052703 (2001).

38 A. V. Avdeenkov J. L. and Bohn, Phys. Rev. Lett. 90, 043006 (2003).

39 A. V. Avdeenkov J. L. and Bohn, Phys. Rev. A 71, 022706 (2005).

40 M. S. Child, Molecular collision theory (Academic Press, London, 1974).

41 D. Secrest, Rotational Excitation I: Quantal Treatment, in Atom-Molecule Collision Theory: A Guide for Experimentalist, R. B. Bernstein editor, Plenum Press, New York (1979), pp 265-299.

42 M. H. Alexander and P. McGuire, J. Chem. Phys. 64, 452 (1976); M. H. Alexander, J. Chem. Phys. 66, 4608 (1977).

43 M. H. Alexander, and D. E. Manolopoulos, J. Chem. Phys. 86, 2044 (1987).

44 J. M. Hutson and S. Green, MOLSCAT ver. 14 (distributed by Collaborative Computational Project 6; Daresbury Laboratory: UK Eng. Phys. Sci. Res. Council) (1994).

45 D. W. Schwenke, J. Chem. Phys. 89, 2076 (1988).

46 S. K. Pogrebnya and D. C. Clary, Chem. Phys. Lett. 363, 523 (2002).

47 W. Bauer, B. Lantzsch, J. P. Tonnies, and K Walaschewski, Chem. Phys. 17, 19 (1976).

48 R. V. Krems (private communication). 


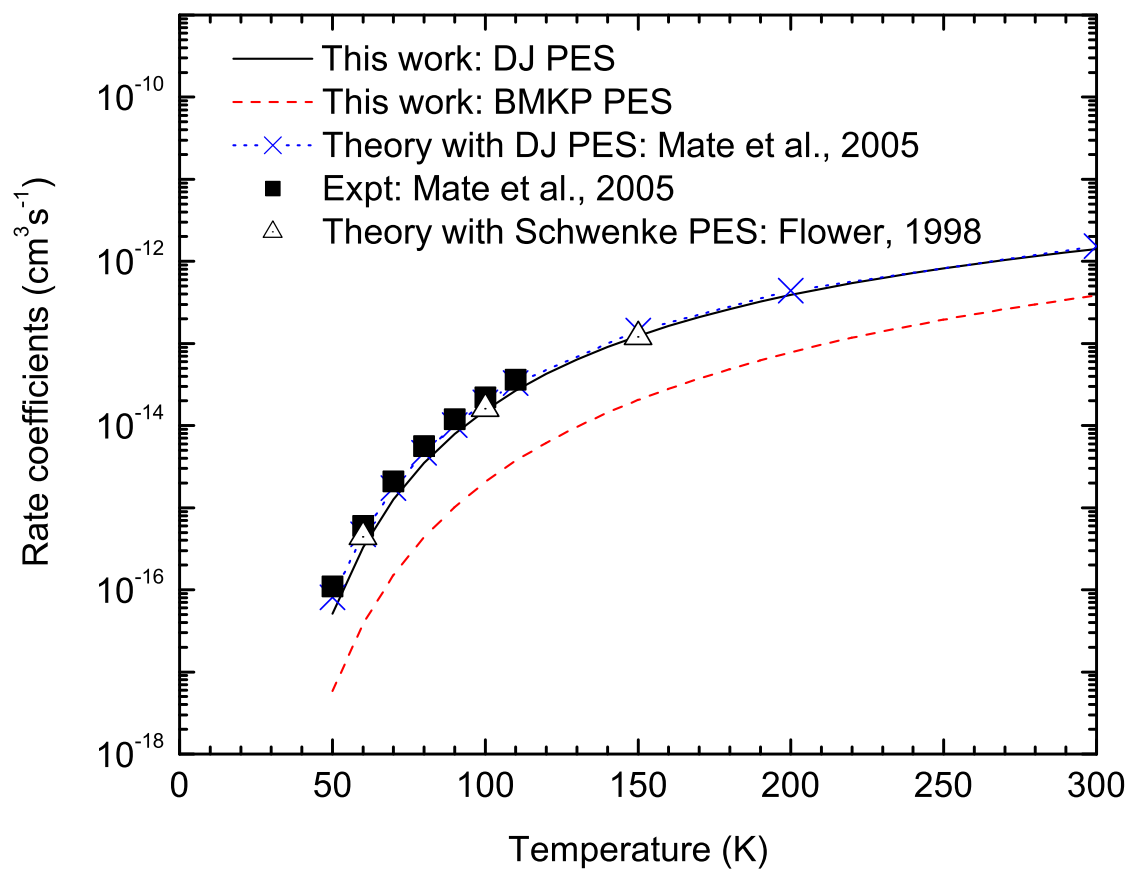

FIG. 1: Rate coefficients for $00 \rightarrow 20$ rotational excitation in $\mathrm{H}_{2}+\mathrm{H}_{2}$ collisions as a function of temperature. The solid and dashed curves are results obtained using the DJ and BMKP potentials, respectively. The solid squares are the experimental results and the dotted line with crosses is the theoretical calculation based on DJ PES by Maté et al.31. The results on the DJ potential agree very well with the experiment data whereas those obtained with BMKP do not. 


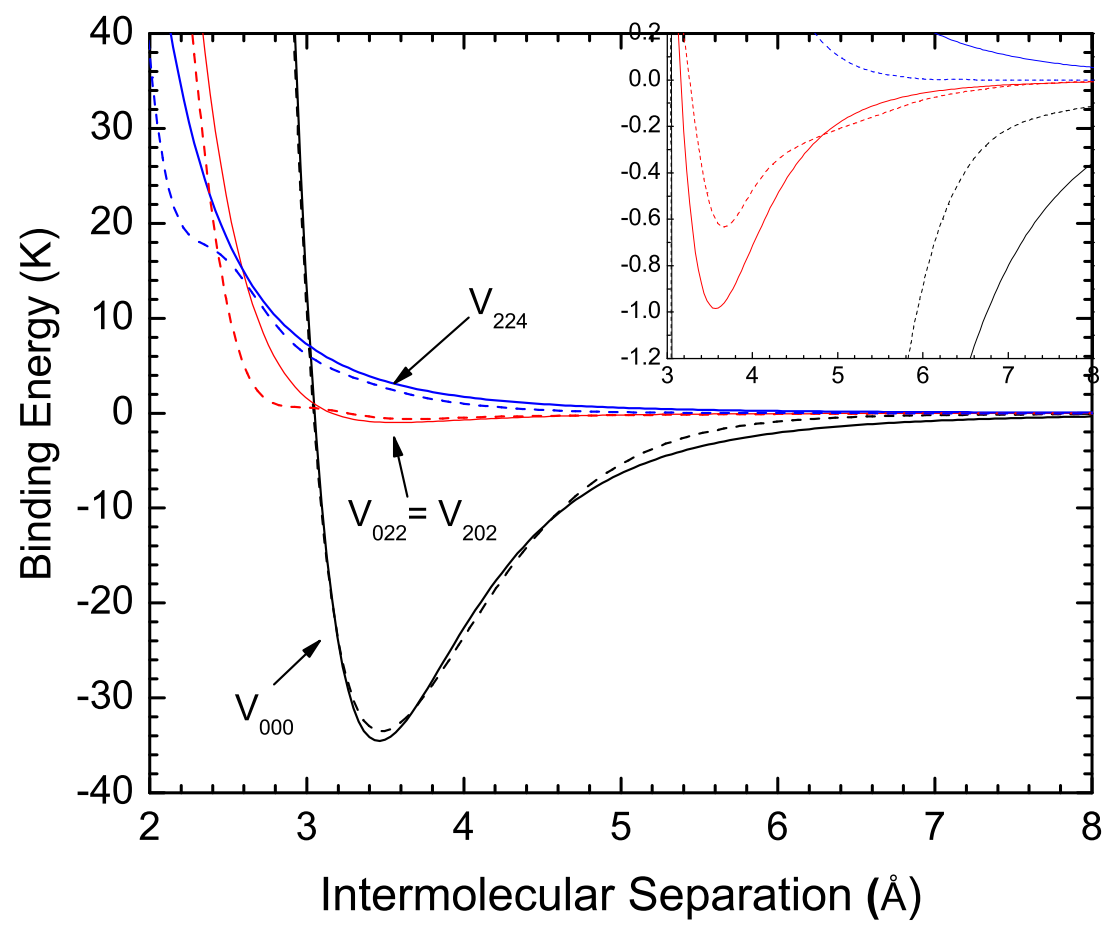

FIG. 2: First three terms in the angular expansion of the intermolecular potential as functions of the intermolecular radial separation. The solid curves represent the DJ potential and the dashed curves denote the BMKP potential. 

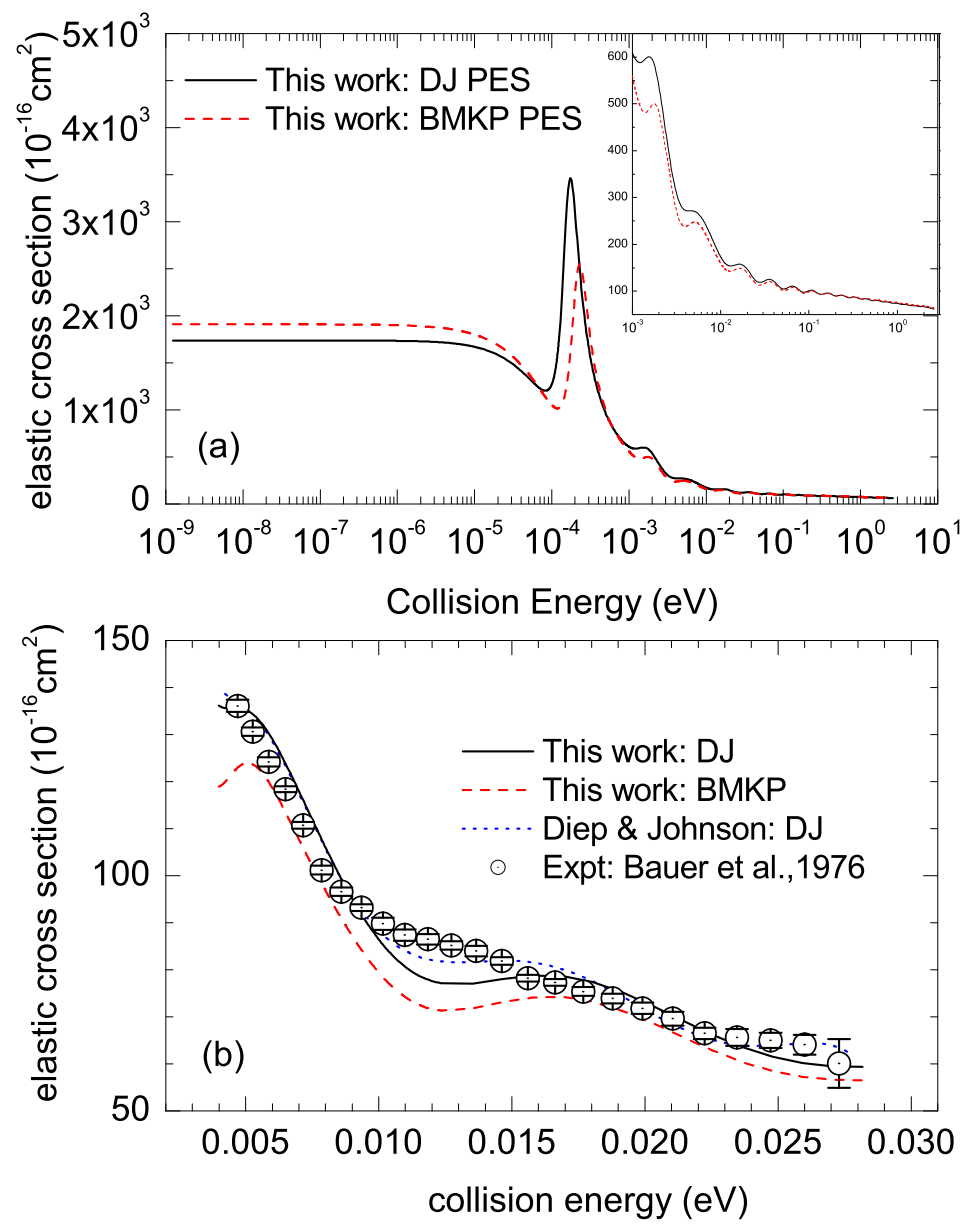

FIG. 3: Elastic cross section $\sigma_{00 \rightarrow 00}$ as a function of collision energy. The solid and dashed curves represent the results on the DJ and BMKP surfaces, respectively. The dotted line is from Diep and Johnson 32 . The circles with error bars are measurements of Bauer et al. $\stackrel{47}{\text {. }}$ 


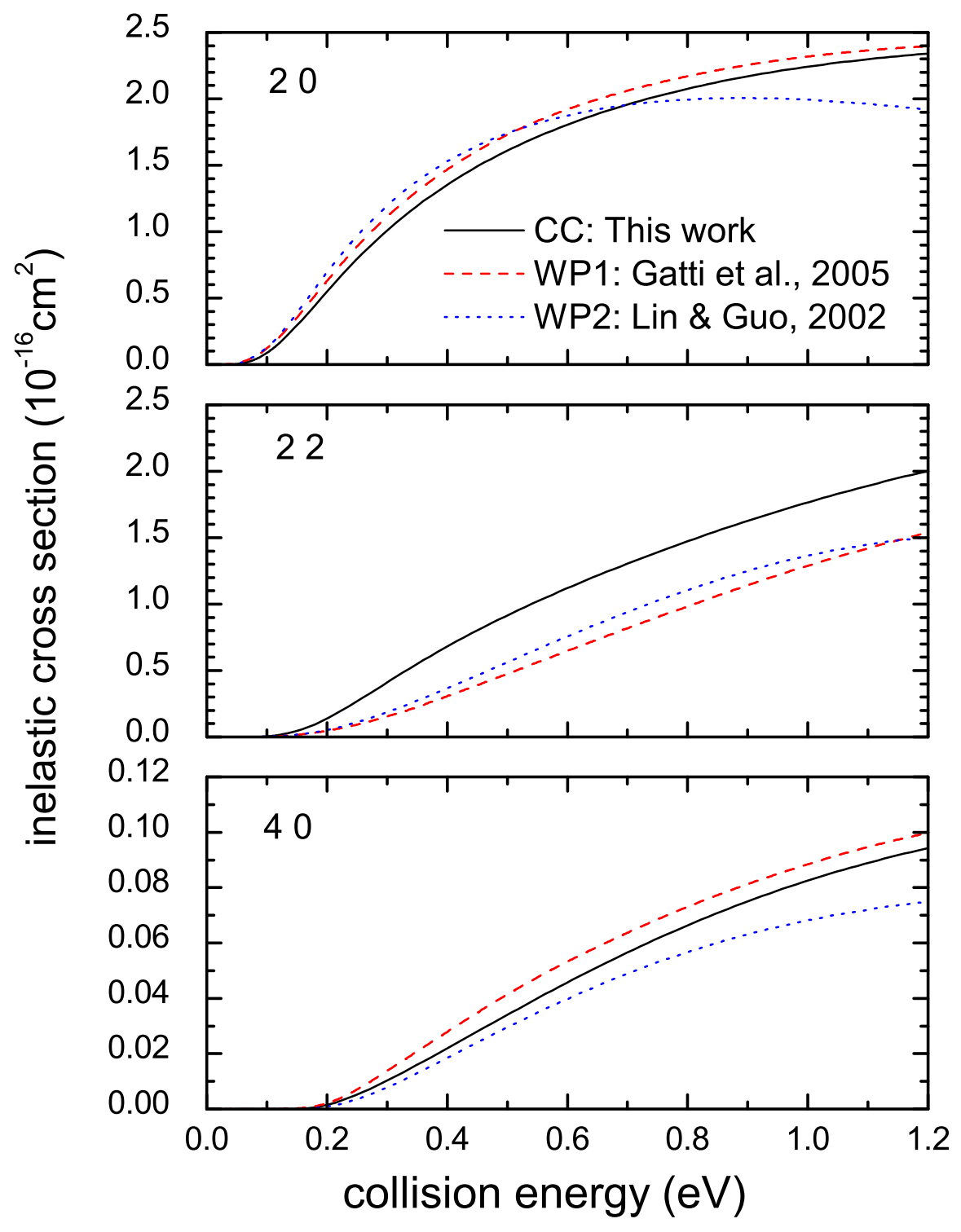

FIG. 4: Comparison of cross sections for $00 \rightarrow 20,22$ and 40 transitions. The calculations are based on the BMKP PES. The solid, dashed and dotted lines are the present close-coupling calculation, wave-packet calculation of Gatti et al. ${ }^{33}$ and Lin and Guc ${ }^{34}$, respectively. 

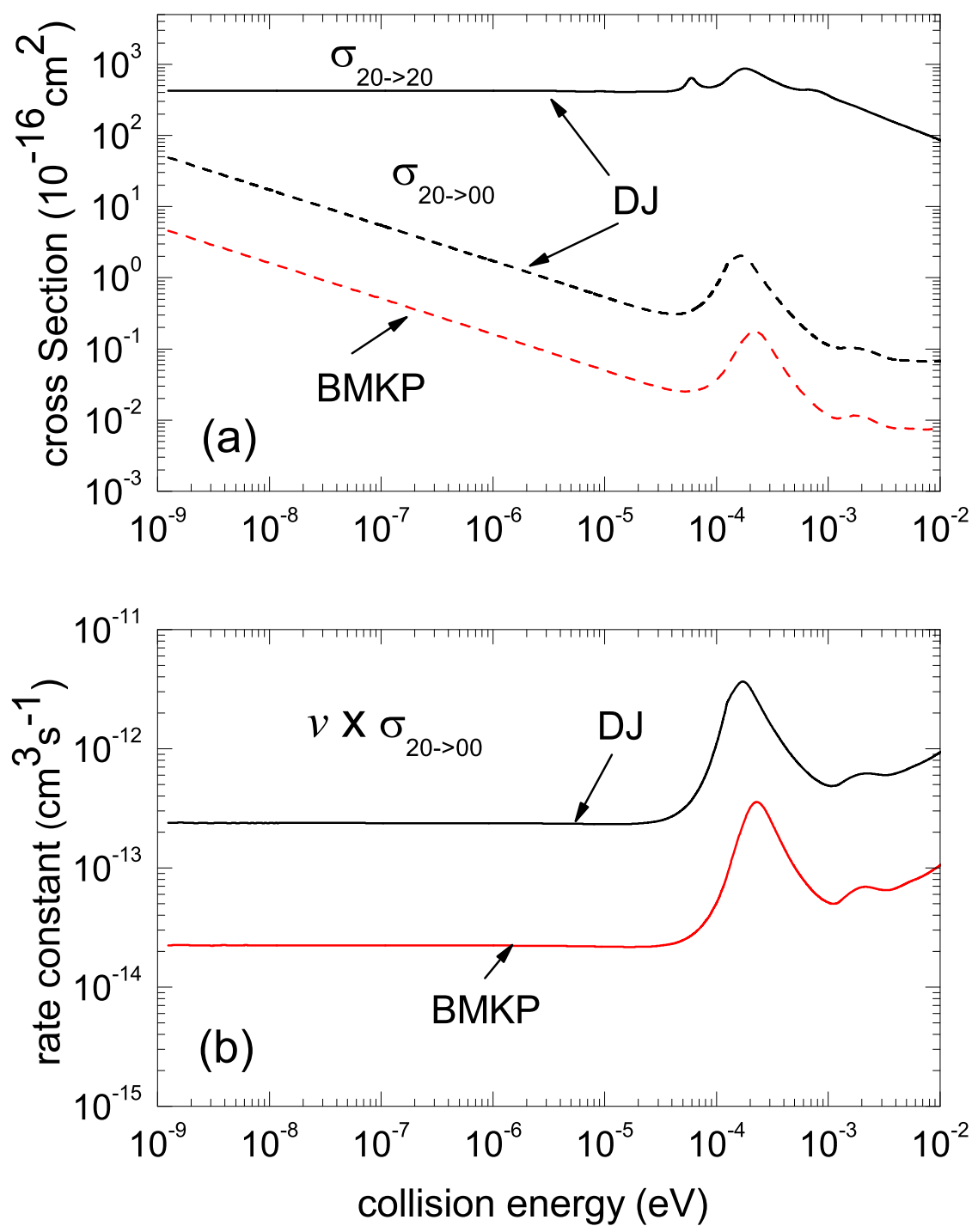

FIG. 5: Cross sections for $20 \rightarrow 00$ rotational quenching as a function of collision energy. (a) Comparison with $20 \rightarrow 20$ elastic cross section. (b) Energy dependent rate coefficients, i.e., relative velocity times cross section. 


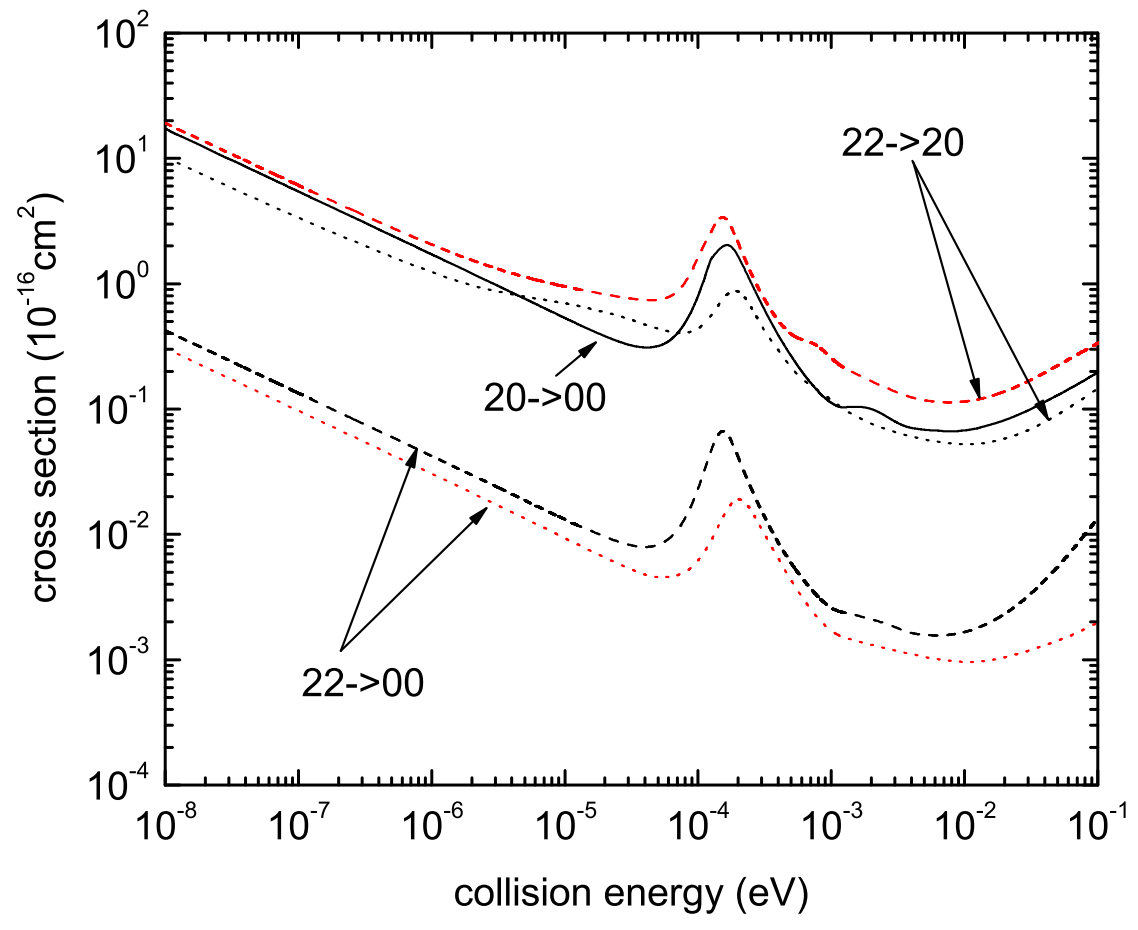

FIG. 6: Cross sections for $22 \rightarrow 00$ and 20 quenching collisions as functions of collision energy plotted against $20 \rightarrow 00$ cross sections. The calculations are based on DJ PES. The dotted lines are the results of Forrey ${ }^{12}$. 


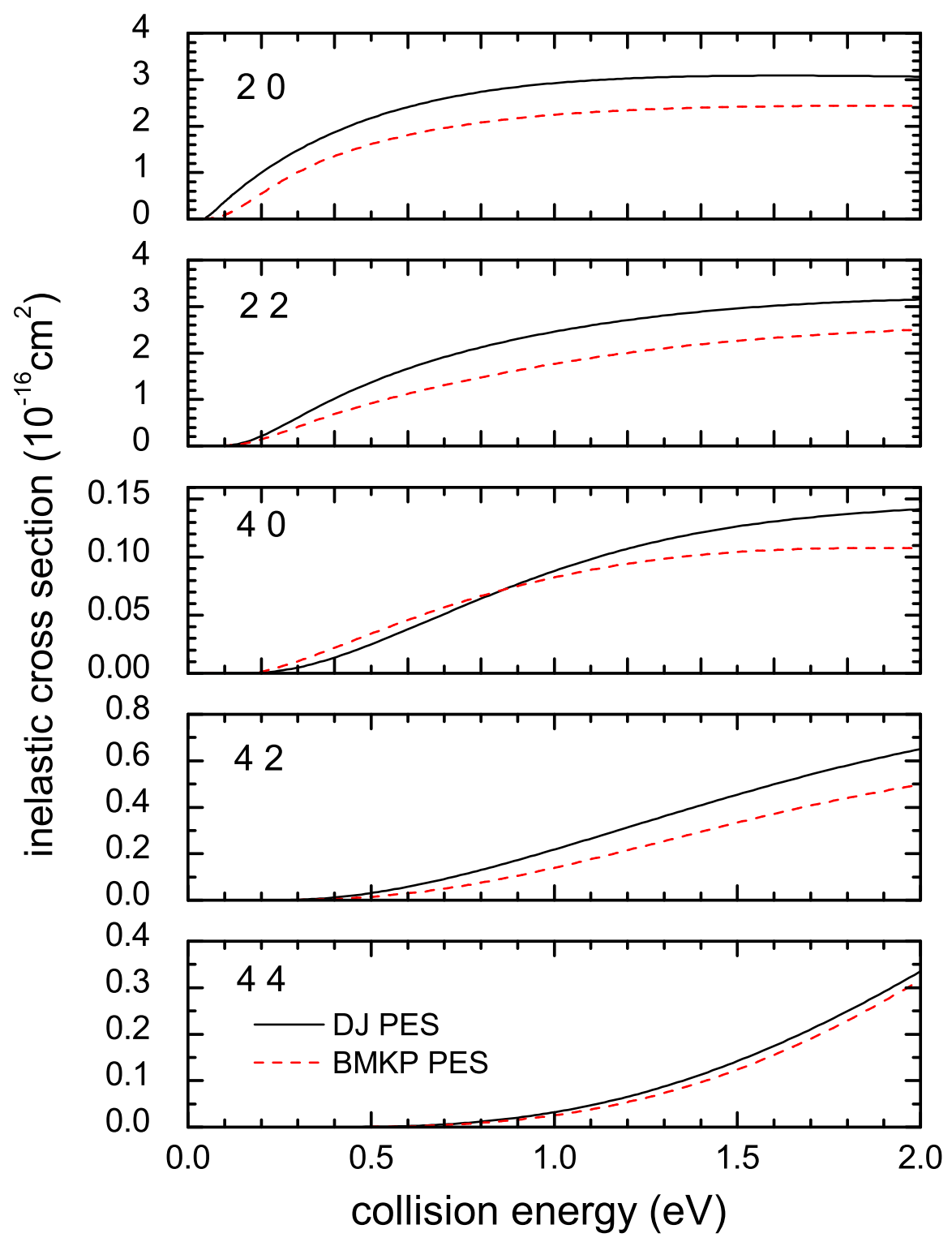

FIG. 7: Comparison of the cross sections for $00 \rightarrow 20,22,40,42$ and 44 transitions as functions of the collision energy. The solid and dashed lines represent DJ and BMKP PESs, respectively. 


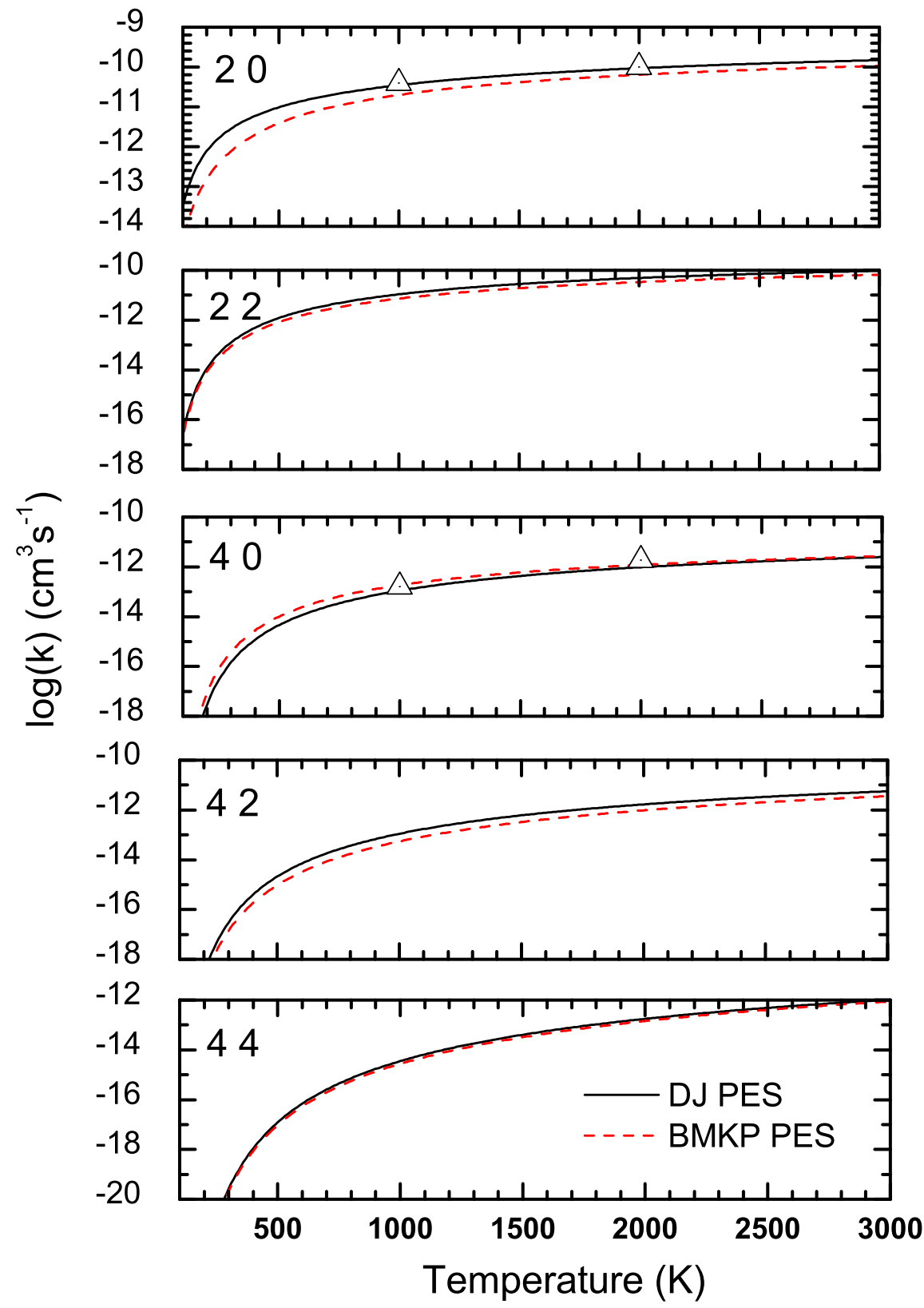

FIG. 8: Similar to Fig. 7, except for the rate coefficient as a function of the temperature. 